\title{
Metal-Free Z-Selective Allylic C-H Nitrogenation, Oxygenation, and Carbonation of Alkenes by Thianthrenation
}

\author{
Ming-Shang Liu, ${ }^{\dagger} \mathrm{Hai}-\mathrm{Wu} \mathrm{Du}^{\dagger}$ and Wei Shu*† \\ †'Shenzhen Grubbs Institute, Department of Chemistry, and Guangdong Provincial Key \\ Laboratory of Catalysis, Southern University of Science and Technology, Shenzhen \\ 518055, Guangdong, P. R. China
}

*E-mail: $\underline{\text { shuw@sustech.edu.cn }}$

\begin{abstract}
Selective functionalization of allylic C-H bonds into other chemical bonds with Z-selectivity are among the most straightforward and attractive, yet challenging transformations. Herein, a transition-metal-free protocol for direct allylic C-H nitrogenation, oxygenation, and carbonation of alkenes by thianthrenation was developed. This operationally simple protocol allows for the unified allylic C-H amination, esterification, etherification, and arylation of vinyl thiathrenium salts. Notably, the reaction preferably provides multialkyl substituted allylic amines, esters, and ethers with Z-selectivity. The reaction proceeds under mild conditions with excellent functional group tolerance and could be applied to late-stage allylation of natural products, drug molecules and peptides with excellent chemoselectivity.
\end{abstract}

Methods for direct allylic $\mathrm{C}-\mathrm{H}$ functionalizations are among the most attractive transformations to streamline organic synthesis as it maximizes the step- and atomeconomy to generate stereodefined allylic species amenable to further chemical transformations, thus minimizing the cost and waste. ${ }^{1-8}$ Traditional allylic C-H functionalization reactions generally require the catalysis of transition-metals such as $\mathrm{Pd}, \mathrm{Cu}$, and $\mathrm{Ir}$, in which involve the formation of an allyl-metal complex via $\mathrm{C}-\mathrm{H}$ activation followed by being attacked by an intra- or intermolecular nucleometallation (Fig. 1a). ${ }^{9-28}$ Recently, the radical cleavage of an allylic C-H bond via hydrogen atom transfer is also developed to generate carbon-centered radical intermediates, which could be involved in following radical processes or transtion-metal catalysis. ${ }^{21,29-35}$ These two strategies proved to be powerful for organic synthesis and extensively investigated. The stereochemistry outcomes heavily rely on the property of transitionmetals and anchoring ligands and are mostly dominated by thermodynamic control, leading to the formation of $\mathrm{C}$-H functionalizaiton products with more stable $E$ selectivity. ${ }^{9-24,36-44}$ On the contrast, the realization of Z-selective allylic C-H functionalizations is more challenging and remains elusive. ${ }^{45-47}$ On the other hands, organothiathrenium salts could be easily prepared from arenes and alkenes by thianthrenation using stoichiometric thianthrene S-oxide or phenoxathiine 10-oxide as the mediators, which could serve as the precousors for both cross-coupling reactions and radical precesses. ${ }^{48,49}$ This two-step strategy arises potential chemical space for 
manipulating $\mathrm{C}-\mathrm{H}$ bond of arenes and alkenes. Recently, Ritter group reported seminal work on selective $\mathrm{C}-\mathrm{H}$ functionalizations of arenes via thianthrenation, providing access to diverse chemical bonds from aryl C-H bonds (Fig. 1b). ${ }^{50-57}$ Wang group developed the Pd-catalyzed site-selective $\mathrm{C}-\mathrm{H}$ borylation and arylation of arenes by employing the same strategy (Fig. 1b). ${ }^{58-60}$ Recently, Shi group developed the utilization of alkyl thianthrenium salts for C-B and C-C bond formation. ${ }^{61,62}$ Wickens group reported the electrochemical synthesis of 1,2-disubstituted thianthrenium salts from alkenes and thianthrene, which were used as the key intermediates to produce aziridines by $\mathrm{C}-\mathrm{N}$ formation. ${ }^{63}$ In 2020, Ritter group developed elegant examples of C$\mathrm{H}$ functionalizations of alkenes via the isolated vinyl thianthrenium salts, giving alkylation, alkynylation, arylation, halogenation, and trifluorosulfinylation of alkenes (Fig. 1b). ${ }^{64,65}$ Despite the promising progress, all the above-mentioned $\mathrm{C}-\mathrm{H}$ functioanlization reactions are restricted to ipso-functionalizations of arenes or alkenes via thianthrenation by replacing the $\mathrm{C}$-S bond in the thiathrenium salts. Yet, no example of $\mathrm{C}-\mathrm{H}$ functionalization at the allylic position of alkenes was reported. Herein, we reported the metal-free selective allylic $\mathrm{C}-\mathrm{H}$ functionalizations of alkenes via thianthrenation (Fig. 1c). The mild conditions allow for the allylic $\mathrm{C}-\mathrm{H}$ nitrogenation, oxygenation, and carbonation of alkenes at room temperature by the formation of C-O, $\mathrm{C}-\mathrm{C}$, and C-N bonds, affording diverse esterifications, thioesterifications, etherifications, aminations, arylation of allylic C-H bonds. ${ }^{66}$ Notably, the metal-free C$\mathrm{H}$ functionalizations deliver allylic esters, ethers, amines, ammonium salts, and amides with preferred Z-selectivity.

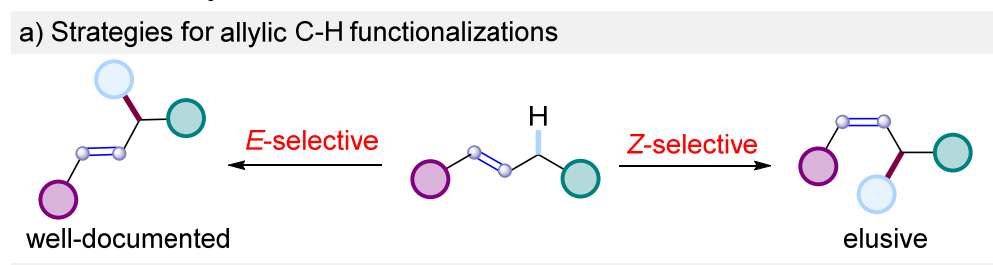

b) Thiathrenium salts: ipso-functionalizations
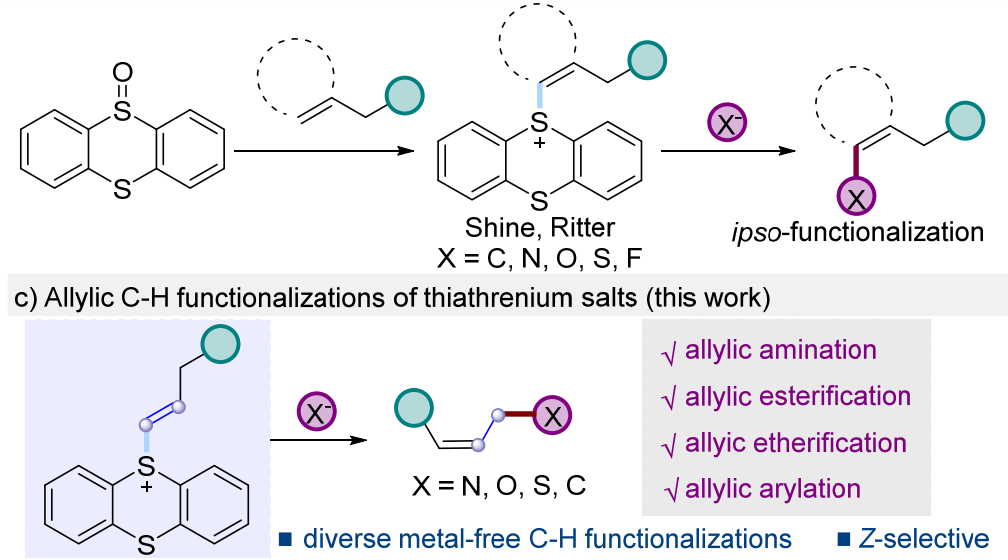

Fig. 1 Impetus for metal-free Z-selective allylic $\mathrm{C}-\mathrm{H}$ functionalizations of thianthrenium salts

We started to investigate the reaction by using cyclohexylvinylthiathrenium salt 1a with benzoic acid 2a. Interestingly, translocation of alkene by allylic C-H functionalization was observed, affording 1,1,2-trialkyl substituted alkene 3a instead of ipso-vinyl substitution of vinylthiathrenium. After evaluation of a variety of reaction 
parameters, we defined the use of potassium carbonate (1.0 equiv) as base in DCM ( 0.1 $\mathrm{M})$ at room temperature as standard conditions, delivering the desired prouct $\mathbf{3 a}$ in $91 \%$ isolated yield (Table 1, entry 1). The use of other bases could also mediate the reaction. Cesium carbonate and potassium phosphate tribasic delivered $\mathbf{3 a}$ in $85 \%$ and $72 \%$ yields, respectively (Table 1, entries 2 and 3). Lithium carbonate led to no formation of 3a (Table 1, entry 4). The reaction proceeded in most tested solvents, including polar and nonpolar solvents, furnishing the desired product $\mathbf{3 a}$ in $47-88 \%$ yields (Table 1, entries 5-9).

Table 1. Condition development of the reaction.

\begin{tabular}{ccc} 
entries $^{[\mathrm{a}]}$ & Variation from "standard conditions" \\
\hline 1 & none & yield of 3a \\
2 & $\mathrm{Cs}_{2} \mathrm{CO}_{3}$ as base & $92 \%(91 \%)^{\mathrm{b}}$ \\
3 & $\mathrm{~K}_{3} \mathrm{PO}_{4}$ as base & $85 \%$ \\
4 & $\mathrm{Li}_{2} \mathrm{CO}_{3}$ as base & $72 \%$ \\
5 & $\mathrm{DCE}$ as solvent & $\mathrm{N} . \mathrm{D}$. \\
6 & $\mathrm{CH}_{3} \mathrm{CN}_{\text {as solvent }}$ & $88 \%$ \\
7 & acetone as solvent & $82 \%$ \\
8 & $\mathrm{THF}$ as solvent & $69 \%$ \\
9 & toluene as solvent & $47 \%$ \\
\hline
\end{tabular}

${ }^{a}$ The reaction was conducted using 1 a $(0.1 \mathrm{mmol})$ and $\mathbf{2 a}(0.12 \mathrm{mmol})$ at room temperature for $24 \mathrm{~h}$. Yield was determined by ${ }^{1} \mathrm{H}$ NMR of the crude mixture using mesitylene as internal standard. ${ }^{b}$ Isolated yield after flash chromatography.

With the optimized conditions established, we turned to evaluate the scope of this reaction. It is found that the reaction conditions tolerate a variety of vinylthiathrenium salts and different nucleophiles with broad functional group and substitution pattern compatibility (Fig. 2). First, the scope for allylic C-H esterification was examined. A surprisingly wide range of carboxylic acids were tolerated (3a-3o). Aromatic and heteroaromatic carboxylic acids, such as quinoline carboxylic acid, thiophene carboxylic acid, indole carboxylic acid, furan carboxylic acid, pyrrole carboxylic acid, could be involved in the reaction to deliver the allylic $\mathrm{C}-\mathrm{H}$ esterification products (3b3f) in $72-83 \%$ yields. Aliphatic acids, including $\alpha$-linear, $\alpha$-branched and $\alpha$-tertiary carboxylic acids, are good substrates for this metal-free $\mathrm{C}-\mathrm{H}$ functionalization process, giving corresponding esters (3g-3k) in $64-89 \%$ yields. Formic acid could form allylic formic ester $\mathbf{3 l}$ in $74 \%$ yield. Benzoylformic acid could form corresponding ester $\mathbf{3 m}$ in $77 \%$ yield via allylic $\mathrm{C}-\mathrm{H}$ functionalization. Propiolic acid was tolerated to give corresponding allylic ester $\mathbf{3 n}$ in $86 \%$ yield. Conjugated dienoic acid was converted to 3o in $90 \%$ yield, leaving the conjugated diene intact. Moreover, this protocol was applicable to late-stage functionalization of complex molecules. Naproxen was transformed to corresponding allylic ester $\mathbf{3 p}$ in $84 \%$ yield without erasing the stereogenic center. Oxaprozin, adapalene, lithocholic acid, telmisartan, D-biotin, and 
probenecid were all good substrates for this allylic $\mathrm{C}-\mathrm{H}$ esterification reaction, furnishing corresponding esters (3q-3v) in $67-95 \%$ yields. Potassium benylpenicillin was compatible in the reaction, delivering the esterification product $\mathbf{3 w}$ in $92 \%$ yield in high chemoselectivity, without detecting the $\mathrm{N}$-allylation product. Notably, the reaction tolerated a wide range of natural and unnatural amino acids and peptides. N-Boc protected L-proline was successfully converted to allylic ester $3 \mathbf{x}$ in $86 \%$ yield. Peptides, such as CBz-Gly-Gly and neotame underwent allylic C-H esterification selectively to leave free amide and amine unreactive, affording $\mathbf{3 y}$ and $\mathbf{3 z}$ in $79 \%$ and $84 \%$ yields, respectively. Next, the scope of vinylthiathrenium salts was investigated. Five, six, seven-membered cyclic alkenes could be involved to undergo allylic $\mathrm{C}-\mathrm{H}$ oxygenation with benzoic acid to furnish cyclic allylic esters (4a-4c) in 56-74\% yields. Cyclododecene was converted to corresponding allylic ester $4 \mathbf{d}$ in $58 \%$ yield with exclusive Z-selectivity. A mixture of isomers of alkene-derived thiathrenium salt delivered a single isomer of the corresponding allylic C-H oxygenation product $4 \mathbf{e}$ in $80 \%$ yield. It is noteworthy that 1 -substituted alkene based thiathrenium salts were converted to allylic esterification products smoothly. Surprisingly, the reaction delivered 1,2-substituted alkenes favored Z-selectivity. Alkenes with pendant bromides, alkenes, alcohols, esters were all compatible in the reaction, delivering the esterification of allylic $\mathrm{C}-\mathrm{H}$ bonds in 56-95\% yields with 2.0:1-3.8:1 ratios of Z-selectivity (4f-4m). Notably, the Z-selectivity could be further improved up to $9.0: 1$ (4g, $\mathbf{4 h}, \mathbf{4 k}$, and $\mathbf{4 m}$ ) using pentamethyldiethylenetriamine (PMDTA) as the base. The configuration of the major product was confirmed unambiguously by X-ray diffraction of $\mathbf{4 m}$. Moreover, the reaction could be easily scaled up. The reaction on $4.0 \mathrm{mmol}$ scale afforded $1.19 \mathrm{~g}$ of $4 \mathrm{~m}$ in $83 \%$ yield. Next, the application of allylic $\mathrm{C}-\mathrm{H}$ functionalization was extended to other nucleophiles was examined. Allylic $\mathrm{C}-\mathrm{H}$ etherification was successful using both alcohols and phenols as the nucleophile, furnishing alkyl and phenyl allylic ethers (5a and 5b) in $45 \%$ and $66 \%$ yields. Thioesterification of allylic $\mathrm{C}-\mathrm{H}$ bond was achieved in $97 \%$ yield (5c) using potassium thioacetate. Allylic C-H arylation was also accomplished in $62 \%$ yield (5d) with trimethoxybenzene as the nucleophile. Moreover, allylic $\mathrm{C}-\mathrm{H}$ amination was also demonstrated. Primary anilines, aliphatic amines were all well tolerated, delivering allylic secondary amines (6a-6c) in 55-72\% yields. Secondary amines with different substitution patterns were all good substrates for this reaction, giving diverse allylic tertiary amines (6d-6g) in 55-71\% yields. Monosubstituted alkene based thiathrenium salts were converted to Z-selective 1,2disubstituted allylic amines in $47-75 \%$ yields with 1.9:1-4.9:1 ratio $(\mathbf{6 h}-\mathbf{6 j})$. The configuration of the major isomer of allylic amines was further confirmed by the X-ray diffraction of the salt of $\mathbf{6 h}$. Impressively, tertiary amines were also compatible in allylic C-H amination reaction to afford allylic trialkyl ammonium salts in $70-76 \%$ yields (6k6m). When 4-phenyl-1-butene derived thiathrenium salt was exposed in the reaction conditions with triethyl amine and quinuclidine, the desired allylic ammonium salts were obtained in $72 \%$ and $70 \%$ yields (6I and $\mathbf{6 m}$ ), favoring Z-selectivity in 4.4:1. Notably, allylic C-H sulfonyl amidation of vinyl thiathrenium salts were also successful, affording corresponding allylic sulfonyl amides in $73 \%$ and $75 \%$ yields (6n and 60 ), respectively. 


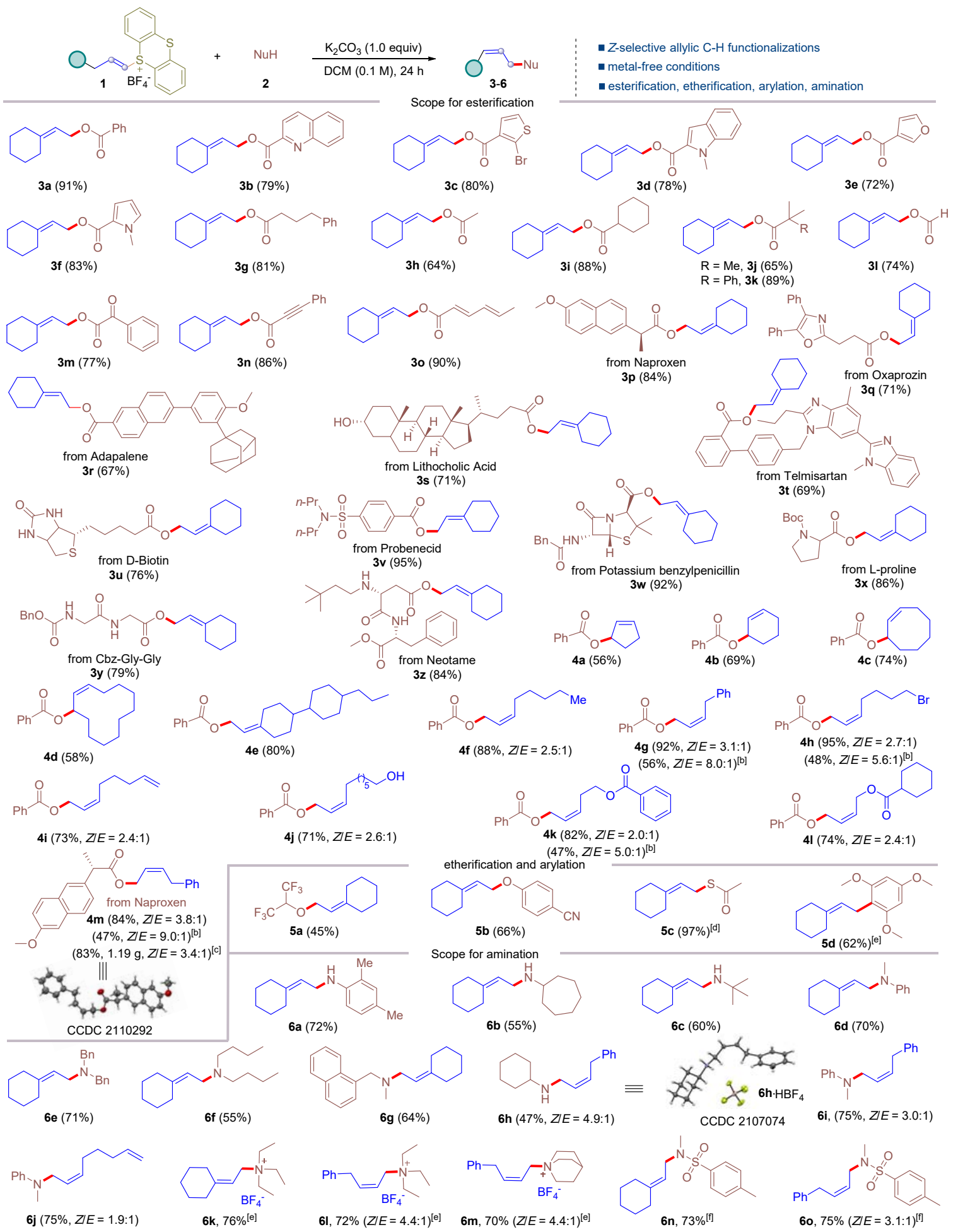

Fig. 2 Scope for the metal-free allylic $\mathrm{C}-\mathrm{H}$ functionalizations of vinyl thianthrenium salts. ${ }^{a}$ The reaction was conducted on $0.2 \mathrm{mmol}$ scale. Standard conditions, see Table 1 for detail. ${ }^{b}$ PMDTA (pentamethyldiethylenetriamine, 1.0 equiv) was used as base with nucleophile (2.0 equiv) in DCE $(0.05 \mathrm{M}) .{ }^{c} 4.0 \mathrm{mmol}$ scale reaction. ${ }^{d}$ Potassium thioacetate and $\mathrm{H}_{2} \mathrm{O}(0.2 \mu \mathrm{L})$ were used. ${ }^{e}$ The reaction was conducted using amine $(2.0$ equiv), $\mathrm{H}_{2} \mathrm{O}(0.2 \mu \mathrm{L}) .{ }^{f} \mathrm{KOH}$ (1.0 equiv) was used as base. 


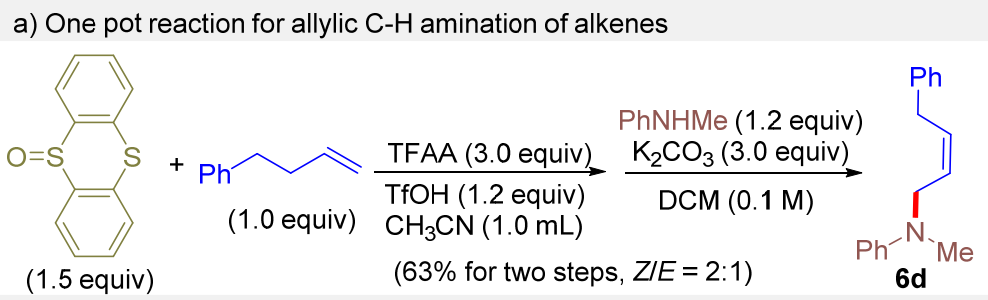

b) Radical probe

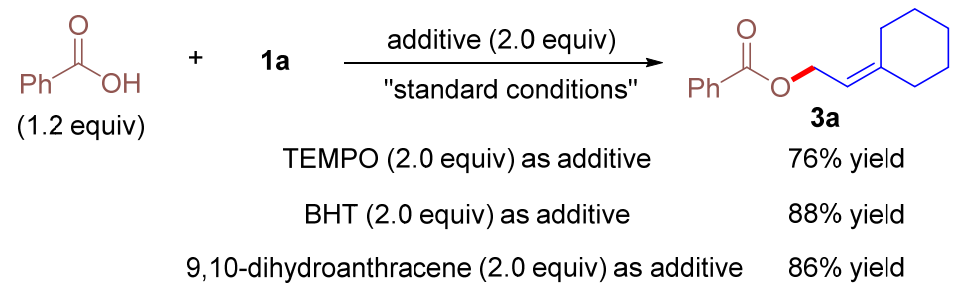

c) Assignment of reaction intermediate

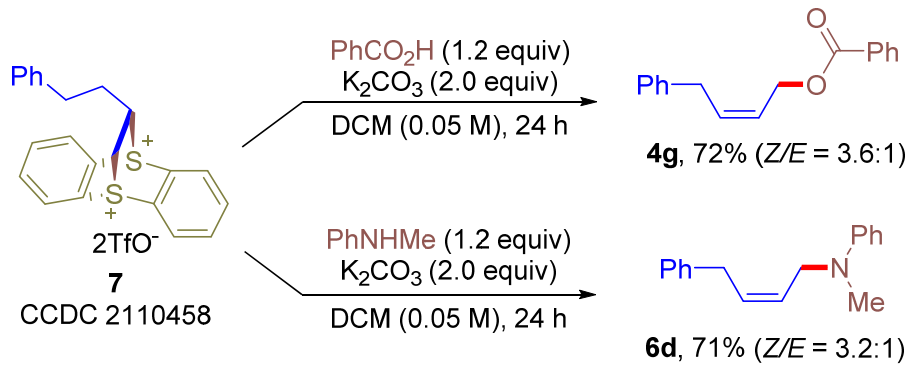

Fig. 3 One-pot synthesis and control experiments.

To demonstrate the practicality of this reaction, a one-pot operation from an alkene and thianthreneoxide, followed by a nucleophile was demonstrated (Fig. 3a). The one pot reaction from 3-phenyl-1-propene, followed by N-methylaniline could afford the desired allylic amine $\mathbf{6 d}$ in $63 \%$ yield without any workup or intermediate purification, which is comparable to previous result. Next, the reaction of 1a with $2 \mathbf{a}$ was conducted in the presence of a radical scavenger under otherwise identical to standard conditions (Fig. 3b). It is shown that the reaction proceeded smoothly in the presence of TEMPO, BHT or 9,10-dihydroanthracene, affording the desired product 3a without erasing the efficacy. These results exclude the involving of radical intermediates in this reaction. To further probe the mechanism of the reaction, a dithianthrenium salt 7 was submitted to the reaction with benzoic acid or $\mathrm{N}$-methylaniline, corresponding allylic $\mathrm{C}-\mathrm{H}$ esterification product $\mathbf{4 g}$ and amination product $\mathbf{6 d}$ were obtained in $72 \%$ and $71 \%$ yield, respectively (Fig. 3c). The yield and stereoselectivity are comparable to the results of using corresponding vinylthianthrenium. These results indicate dithianthrenium salt could serve as the reactive intermediate for this reaction.

Based on literature and the experimental results, a plausible mechanism is described in Fig. 4. First, intramolecular attack of sulfur on alkene moiety of vinylthianthrenium salt could deliver the dithianthrenium salt M1, which could further undergo site-selective ring-opening by intermolecular attack by a nucleophile to give an alkylthianthrenium salt intermediate M2. In the presence of a base, M2 would undergo a syn-elimination via TS1 to give the final allylic $\mathrm{C}-\mathrm{H}$ functionalization product in favor of Z-selectivity. 


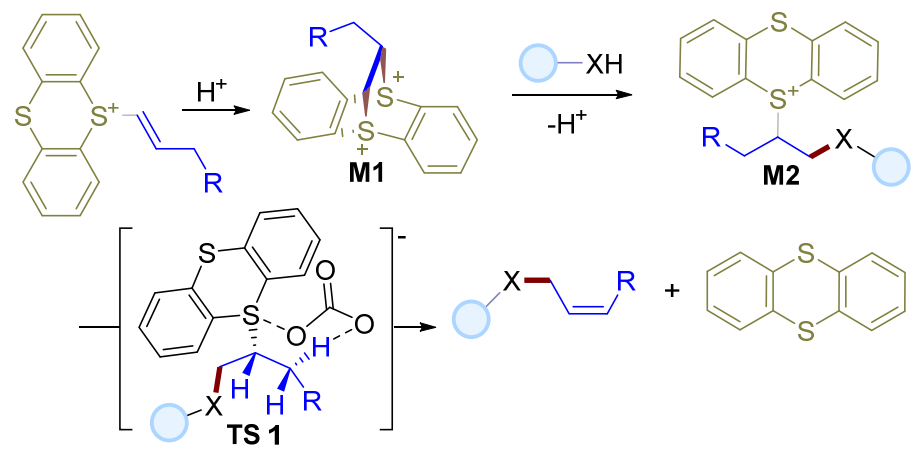

Fig. 4 Proposed mechanism for the reaction.

In summary, a unified transition-metal-free protocol for diverse functionalizations of allylic $\mathrm{C}-\mathrm{H}$ bonds of alkenes by thianthrenation under mild conditions has been demonstrated for the first time. Notably, the reaction features Z-selectivity to afford multi-alkyl substituted allylic esters, thioesters, ethers, primary, secondary, tertiary amines, amides, and arenes in good yields without incorporation of any transitionmetals. One-pot procedure proved efficient to access direct allylic C-H functionalizations from alkenes. The reaction tolerates a wide range of O-, Nnucleophiles with excellent functional group tolerance, and could be applied to latestage functionalizations of natural products, amino acids, and drug-like molecules with excellent chemoselectity.

\section{Acknowledgements}

We sincerely acknowledge NSFC (21971101, 21801126, 22171127), Thousand Talents Program for Young Scholars, Guangdong Basic and Applied Basic Research Foundation (2019A1515011976), Department of Education of Guangdong Province (2021KTSCX06), The Stable Support Plan Program of Shenzhen Natural Science Fund (No. 20200925152608001), The Pearl River Talent Recruitment Program (2019QN01Y261), Guangdong Provincial Key Laboratory of Catalysis (No. 2020B121201002) for financial support. We acknowledge the assistance of SUSTech Core Research Facilities. We thank Dr. Xiaoyong Chang (SUSTech) for X-ray crystallographic analysis (CCDC 2110292, 2107074, 2110458) and Bi-Hong Chen (SUSTech) for reproducing the results of $\mathbf{3 g}, \mathbf{4 f}, \mathbf{6} \mathbf{a}$ and $\mathbf{6 i}$.

\section{References:}

1. Eames, J. \& Watkinson, M. Catalytic Allylic Oxidation of Alkenes Using an Asymmetric Kharasch-Sosnovsky Reaction. Angew. Chem. Int. Ed. 40, 3567-3571 (2001).

2. Adam, W. \& Krebs, O. The Nitroso Ene Reaction: A Regioselective and Stereoselective Allylic Nitrogen Functionalization of Mechanistic Delight and Synthetic Potential. Chem. Rev. 103, 4131-4146 (2003).

3. Ramirez, T. A., Zhao, B. \& Shi, Y. Recent Advances in Transition Metal-Catalyzed $\mathrm{sp}^{3} \mathrm{C}-\mathrm{H}$ Amination Adjacent to Double Bonds and Carbonyl Groups. Chem. Soc. Rev. 41, 931-942 (2012).

4. Zheng, C. \& You, S.-L. Recent Development of Direct Asymmetric Functionalization 
of Inert C-H Bonds. RSC Adv. 4, 6173-6214 (2014).

5. Bayeh, L. \& Tambar, U. K. Catalytic Asymmetric Intermolecular Allylic Functionalization of Unactivated Internal Alkenes. ACS Catal. 7, 8533-8543 (2017).

6. Kazerouni, A. M., McKoy, Q. A. \& Blakey, S. B. Recent Advances in Oxidative Allylic C-H Functionalization via Group IX-metal Catalysis. Chem. Commun. 56, 13287-13300 (2020).

7. Wang, P. S. \& Gong, L. Z. Palladium-Catalyzed Asymmetric Allylic C-H Functionalization: Mechanism, Stereo- and Regioselectivities, and Synthetic Applications. Acc. Chem. Res. 53, 2841-2854 (2020).

8. Dyker, G. in Handbook of C-H Transformations, Wiley-VCH, Weinheim, 2005.

9. Larock, R. C., Hightower, T. R., Hasvold, L. A. \& Peterson, K. P. Palladium(II)Catalyzed Cyclization of Olefinic Tosylamides. J. Org. Chem. 61, 3584-3585 (1996).

10. Franzen, J. \& Backvall, J.-E. Carbon-Carbon Bond Formation in Palladium(II)Catalyzed Allylic Oxidation: A Novel Oxidative Carbocyclization of AlleneSubstituted Olefins. J. Am. Chem. Soc. 125, 6056-6057 (2003).

11. Chen, M. S. \& White, M. C. A Sulfoxide-Promoted, Catalytic Method for the Regioselective Synthesis of Allylic Acetates from Monosubstituted Olefins via C-H Oxidation. J. Am. Chem. Soc. 126, 1346-1347 (2004).

12. Fraunhoffer, K. J. \& White, M. C. syn-1,2-Amino Alcohols via Diastereoselective Allylic C-H Amination. J. Am. Chem. Soc. 129, 7274-7276 (2007).

13. Liu, G., Yin, G. \& Wu, L. Palladium-Catalyzed Intermolecular Aerobic Oxidative Amination of Terminal Alkenes: Efficient Synthesis of Linear Allylamine Derivatives. Angew. Chem. Int. Ed. 47, 4733-4736 (2008).

14. Reed, S. A. \& White, M. C. Catalytic Intermolecular Linear Allylic C-H Amination via Heterobimetallic Catalysis. J. Am. Chem. Soc. 130, 3316-3318 (2008).

15. Lin, S., Song, C.-X., Cai, G.-X., Wang, W.-H. \& Shi, Z.-J. Intra/Intermolecular Direct Allylic Alkylation via Pd(II)-Catalyzed Allylic C-H Activation. J. Am. Chem. Soc. 130, 12901-12903 (2008).

16. Young, A. J. \& White, M. C. Catalytic Intermolecular Allylic C-H Alkylation. J. Am. Chem. Soc. 130, 14090-14091 (2008).

17. Vermeulen, N. A., Delcamp, J. H. \& White, M. C. Synthesis of Complex Allylic Esters via C-H Oxidation vs C-C Bond Formation. J. Am. Chem. Soc. 132, 11323-11328 (2010).

18. Campbell, A. N., White, P. B., Guzei, I. A. \& Stahl, S. S. Allylic C-H Acetoxylation with a 4,5-Diazafluorenone-Ligated Palladium Catalyst: A Ligand-Based Strategy To Achieve Aerobic Catalytic Turnover. J. Am. Chem. Soc. 132, 15116-15119 (2010).

19. Wang, G.-W., Zhou, A.-X., Li, S.-X. \& Yang, S.-D. Regio- and Stereoselective Allylic C-H Arylation with Electron-Deficient Arenes by 1,1'-Bi-2-naphthol-Palladium Cooperation. Org. Lett. 16, 3118-3121 (2014).

20. Tran, B. L., Driess, M. \& Hartwig, J. F. Copper-Catalyzed Oxidative Dehydrogenative Carboxylation of Unactivated Alkanes to Allylic Esters via Alkenes. J. Am. Chem. Soc. 136, 17292-17301 (2014).

21. Yi, X. \& Hu, X. Intermolecular Oxidative Amination of Unactivated Alkenes by Dual Photoredox and Copper Catalysis. Chem. Sci. 12,1901-1906 (2021). 
22. Parsons, A. T. \& Buchwald, S. L. Copper-Catalyzed Trifluoromethylation of Unactivated Olefins. Angew. Chem. Int. Ed. 50, 9120-9123 (2011).

23. Xu, J., Fu, Y., Luo, D.-F., Jiang, Y.-Y., Xiao, B., Liu, Z.-J., Gong, T.-J. \& Liu, L. Copper-Catalyzed Trifluoromethylation of Terminal Alkenes through Allylic C-H Bond Activation. J. Am. Chem. Soc. 133, 15300-15303 (2011).

24. Wang, X., Ye, Y., Zhang, S., Feng, J., Xu, Y., Zhang, Y. \& Wang, J. CopperCatalyzed $\mathrm{C}\left(\mathrm{sp}^{3}\right)-\mathrm{C}\left(\mathrm{sp}^{3}\right)$ Bond Formation Using a Hypervalent Iodine Reagent: An Efficient Allylic Trifluoromethylation. J. Am. Chem. Soc. 133, 16410-16413 (2011).

25. Knecht, T., Mondal, S., Ye, J.-H., Das, M. \& Glorius, F. Intermolecular, BranchSelective, and Redox-Neutral Cp*Ir III-Catalyzed Allylic C-H Amidation. Angew. Chem. Int. Ed. 58, 7117-7121 (2019).

26. Lei, H. \& Rovis, T. Ir-Catalyzed Intermolecular Branch-Selective Allylic C-H Amidation of Unactivated Terminal Olefins. J. Am. Chem. Soc. 141, 2268-2273 (2019). 27. Li, Q. \& Yu, Z.-X. Conjugated Diene-Assisted Allylic C-H Bond Activation: Cationic Rh(I)-Catalyzed Syntheses of Polysubstituted Tetrahydropyrroles, Tetrahydrofurans, and Cyclopentanes from Ene-2-Dienes. J. Am. Chem. Soc. 132, 4542-4543 (2010).

28. Sekine, M., Ilies, L. \& Nakamura, E. Iron-Catalyzed Allylic Arylation of Olefins via $\mathrm{C}\left(\mathrm{sp}^{3}\right)-\mathrm{H}$ Activation under Mild Conditions. Org. Lett. 15, 714-717 (2013).

29. Zhang, X., Wang, M., Li, P. \& Wang, L. n-Bu4NI/TBHP-Catalyzed Direct Amination of Allylic and Benzylic $\mathrm{C}\left(\mathrm{sp}^{3}\right)-\mathrm{H}$ with Anilines Under Metal-Free Conditions. Chem. Commun. 50, 8006-8009 (2014).

30. Cuthbertson, J. D. \& MacMillan, D. W. C. The Direct Arylation of Allylic $s p^{3}$ C-H Bonds via Organic and Photoredox Catalysis. Nature 519, 74-77 (2015).

31. Zhou, R., Liu, H., Tao, H., Yu, X. \& Wu, J. Metal-Free Direct Alkylation of Unfunctionalized Allylic/Benzylic $\mathrm{sp}^{3} \mathrm{C}-\mathrm{H}$ Bonds via Photoredox Induced Radical Cation Deprotonation. Chem. Sci. 8, 4654-4659 (2017).

32. Huang, L. \& Rueping, M. Direct Cross-Coupling of Allylic C( $\left.\mathrm{sp}^{3}\right)-\mathrm{H}$ Bonds with Aryl- and Vinylbromides by Combined Nickel and Visible-Light Catalysis. Angew. Chem. Int. Ed. 57, 10333-10337 (2018).

33. Schwarz, J. L., Schafers, F., Tlahuext-Aca, A., Luckemeier, L. \& Glorius, F. Diastereoselective Allylation of Aldehydes by Dual Photoredox and Chromium Catalysis. J. Am. Chem. Soc. 140, 12705-12709 (2018).

34. Li, J., Zhang, Z., Wu, L., Zhang, W., Chen, P. Lin, Z. \& Liu, G. Site-Specific Allylic C-H Bond Functionalization with a Copper-Bound N-Centred Radical. Nature 574, 516-521 (2019).

35. Huang, C., Ci, R.-N., Qiao, J., Wang, X.-Z., Feng, K., Chen, B., Tung, C.-H. \& Wu, L.-Z. Direct Allylic $\mathrm{C}\left(\mathrm{sp}^{3}\right)-\mathrm{H}$ and Vinylic $\mathrm{C}\left(\mathrm{sp}^{2}\right)-\mathrm{H}$ Thiolation with Hydrogen Evolution by Quantum Dots and Visible Light. Angew. Chem. Int. Ed. 60, 11779-11783 (2021).

36. Sharpless, K. B., Hori, T., Truesdale, L. K. \& Dietrich, C. O. Allylic Amination of Olefins and Acetylenes by Imido Selenium Compounds. J. Am. Chem. Soc. 98, 269271 (1976).

37. Yamanaka, H., Matsuo, J.-i., Kawana, A. \& Mukaiyama, T., A Convenient Method 
for the Synthesis of $\beta, \gamma$-Unsaturated Amines from Alkenes via $\alpha, \beta$-Unsaturated Diphenylsulfonium Salts. Chem. Lett. 32, 626-627 (2003).

38. Bao, H. \& Tambar, U. K. Catalytic Enantioselective Allylic Amination of Unactivated Terminal Olefins via an Ene Reaction/[2,3]-Rearrangement. J. Am. Chem. Soc. 134, 18495-18498 (2012).

39. Souto, J. A., Zian, D. \& Muñiz, K. Iodine(III)-Mediated Intermolecular Allylic Amination under Metal-Free Conditions. J. Am. Chem. Soc. 134, 7242-7245 (2012).

40. Trenner, J., Depken, C., Weber, T. \& Breder, A. Direct Oxidative Allylic and Vinylic Amination of Alkenes through Selenium Catalysis. Angew. Chem. Int. Ed. 52, 8952-8956 (2013).

41. Zhang, Z., Du, H., Xu, J. \& Li, P. Anti-Markovnikov Rearrangement in Sulfur Mediated Allylic C-H Amination of Olefins. Chem. Commun. 52, 11547-11550 (2016). 42. Bayeh, L., Le, P. Q. \& Tambar, U. K. Catalytic Allylic Oxidation of Internal Alkenes to a Multifunctional Chiral Building Block. Nature 547, 196-200 (2017).

43. Liu, W., Ali, S. Z., Ammann, S. E. \& White, M. C. Asymmetric Allylic C-H Alkylation via Palladium(II)/cis-ArSOX Catalysis. J. Am. Chem. Soc. 140, 1065810662 (2018).

44. Lei, H. \& Rovis, T. A Site-Selective Amination Catalyst Discriminates between Nearly Identical C-H Bonds of Unsymmetrical Disubstituted Alkenes. Nat. Chem. 12, 725-731 (2020).

45. Lin, H.-C., Xie, P.-P., Dai, Z.-Y., Zhang, S.-Q., Wang, P.-S., Chen, Y.-G., Wang, T.C., Hong, X. \& Gong, L.-Z. Nucleophile-Dependent Z/E- and Regioselectivity in the Palladium-Catalyzed Asymmetric Allylic C-H Alkylation of 1,4-Dienes. J. Am. Chem. Soc. 141, 5824-5834 (2019).

46. Wang, T.-C., Wang, P.-S. \& Gong, L.-Z. Palladium-Catalyzed Asymmetric Allylic C-H Alkylation of 1,4-Dienes and Glycine Schiff Bases. Sci. China Chem. 63, 454-459 (2020).

47. Dai, Z. Y., Wang, P. S. \& Gong, L. Z. Access to Chiral $\gamma$-Butenolides via PalladiumCatalyzed Asymmetric Allylic C-H Alkylation of 1,4-Dienes. Chem. Commun. 57, 6748-6751 (2021).

48. Silber, J. J. \& Shine, H. J. Ion radicals. XXII. Reaction of Thianthrenium Perchlorate $\left(\mathrm{C}_{12} \mathrm{H}_{8} \mathrm{~S}_{2}{ }^{+} \mathrm{ClO}_{4}{ }^{-}\right)$with Aromatics. J. Org. Chem. 36, 2923-2926 (1971).

49. Qian, D. Q., Shine, H. J., Guzman-Jimenez, I. Y., Thurston, J. H. \& Whitmire, K. H. Mono- and Bisadducts from the Addition of Thianthrene Cation Radical Salts to Cycloalkenes and Alkenes. J. Org. Chem. 67, 4030-4039 (2002).

50. Berger, F., Plutschack, M. B., Riegger, J., Yu, W., Speicher, S., Ho, M., Frank, N.

\& Ritter, T. Site-selective and Versatile Aromatic C-H Functionalization by Thianthrenation. Nature 567, 223-228 (2019).

51. Engl, P. S., Haring, A. P., Berger, F., Berger, G., Perez-Bitrian, A. \& Ritter, T. C-N Cross-Couplings for Site-Selective Late-Stage Diversification via Aryl Sulfonium Salts. J. Am. Chem. Soc. 141, 13346-13351 (2019).

52. Sang, R., Korkis, S. E., Su, W., Ye, F., Engl, P. S., Berger, F. \& Ritter, T. SiteSelective C-H Oxygenation via Aryl Sulfonium Salts. Angew. Chem. Int. Ed. 58, 1616116166 (2019). 
53. Li, J., Chen, J., Sang, R., Ham, W. S., Plutschack, M. B., Berger, F., Chabbra, S., Schnegg, A., Genicot, C. \& Ritter, T. Photoredox Catalysis with Aryl Sulfonium Salts Enables Site-Selective Late-Stage Fluorination. Nat. Chem. 12, 56-62 (2020).

54. Alvarez, E. M., Karl, T., Berger, F., Torkowski, L. \& Ritter, T. Late-Stage Heteroarylation of Hetero(aryl)sulfonium Salts Activated by $\alpha$-Amino Alkyl Radicals. Angew. Chem. Int. Ed. 60, 13609-13613 (2021).

55. Jia, H., Haring, A. P., Berger, F., Zhang, L. \& Ritter, T. Trifluoromethyl Thianthrenium Triflate: A Readily Available Trifluoromethylating Reagent with Formal $\mathrm{CF}_{3}{ }^{+}, \mathrm{CF}_{3}{ }^{*}$, and $\mathrm{CF}_{3}{ }^{-}$Reactivity. J. Am. Chem. Soc. 143, 7623-7628 (2021).

56. Lansbergen, B., Granatino, P. \& Ritter, T. Site-Selective C-H alkylation of Complex Arenes by a Two-Step Aryl Thianthrenation-Reductive Alkylation Sequence. J. Am. Chem. Soc. 143, 7909-7914 (2021).

57. Juliá, F., Shao, Q., Duan, M., Plutschack, M. B., Berger, F., Meteos, J., Lu, C., Xue, X.-S., Houk, K. N. \& Ritter, T. High Site Selectivity in Electrophilic Aromatic Substitutions: Mechanism of C-H Thianthrenation. J. Am. Chem. Soc. 143, 1604116054 (2021).

58. Wu, J., Wang, Z., Chen, X.-Y., Wu, Y., Wang, D., Peng, Q. \& Wang, P. paraSelective Borylation of Monosubstituted Benzenes Using a Transient Mediator. Sci. China Chem. 63, 336-340 (2020).

59. Chen, X.-Y., Huang, Y.-H., Zhou, J. \& Wang, P. Pd-Catalyzed Site-Selective Borylation of Simple Arenes via Thianthrenation. Chin. J. Chem. 38, 1269-1272 (2020). 60. Chen, X.-Y., Nie, X.-X., Wu, Y. \& Wang, P. para-Selective Arylation and Alkenylation of Monosubstituted Arenes Using Thianthrene S-Oxide as a Transient Mediator. Chem. Commun. 56, 5058-5061 (2020).

61. Chen, C., Wang, Z.-J., Lu, H., Zhao, Y. \& Shi, Z. Generation of Non-Stabilized Alkyl Radicals from Thianthrenium Salts for C-B and C-C Bond Formation. Nat. Commun. 12, 4526 (2021).

62. Chen, C., Wang, M., Lu, H., Zhao, B. \& Shi, Z. Enabling the Use of Alkyl Thianthrenium Salts in Cross-Coupling Reactions by Copper Catalysis. Angew. Chem. Int. Ed. 60, 21756-21760 (2021).

63. Holst, D. E., Wang, D. J., Kim, M. J., Guzei, I. A. \& Wickens, Z. K. Aziridine Synthesis by Coupling Amines and Alkenes via an Electrogenerated Dication. Nature 596, 74-79 (2021).

64. Chen, J., Li, J., Plutschack, M. B., Berger, F. \& Ritter, T. Regio- and Stereoselective Thianthrenation of Olefins To Access Versatile Alkenyl Electrophiles. Angew. Chem. Int. Ed. 59, 5616-5620 (2020).

65. Juliá, F., Yan, J., Paulus, F. \& Ritter, T. Vinyl Thianthrenium Tetrafluoroborate: A Practical and Versatile Vinylating Reagent Made from Ethylene. J. Am. Chem. Soc. 143, 12992-12998 (2021).

66. Cheng, Q., Chen, J., Lin, S. \& Ritter, T. Allylic Amination of Alkenes with Iminothianthrenes to Afford Alkyl Allylamines. J. Am. Chem. Soc. 142, 17287-17293 (2020). 
TOC and Abstract

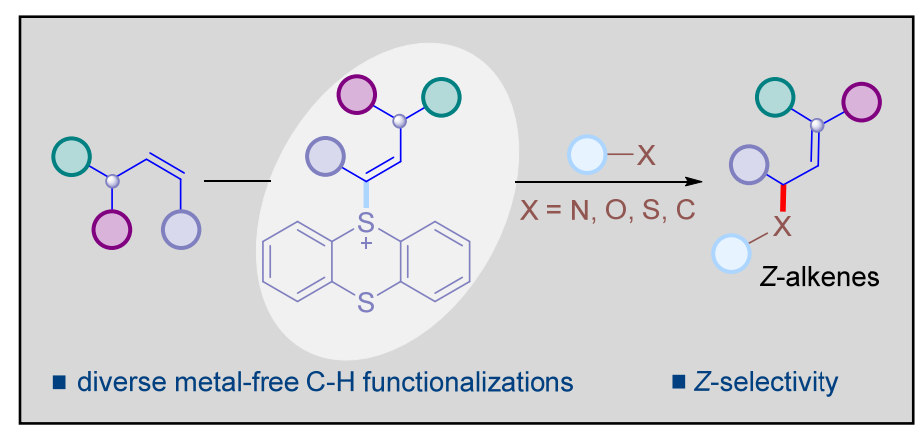

Selective functionalization of allylic $\mathrm{C}-\mathrm{H}$ bonds into other chemical bonds with Zselectivity are among the most straightforward and attractive, yet challenging transformations. Herein, a transition-metal-free protocol for direct allylic C-H nitrogenation, oxygenation, and carbonation of alkenes by thianthrenation was developed. This operationally simple protocol allows for the unified allylic C-H amination, esterification, etherification, and arylation of vinyl thiathrenium salts. Notably, the reaction preferably provides multialkyl substituted allylic amines, esters, and ethers with Z-selectivity. The reaction proceeds under mild conditions with excellent functional group tolerance and could be applied to late-stage allylation of natural products, drug molecules and peptides with excellent chemoselectivity. 